\section{Hepatitis C früher heilen}

Seit kurzem stehen nun wirksame Hepatitis-CTherapien auf der Grundlage von DAA (direct acting antiviral drugs) zur Verfügung. Sie erreichen bei stark verkürzter Behandlungszeit eine Heilungsrate von über $90 \%$. Wegen der hohen Kosten von ca. 60000 CHF pro Behandlung werden die Medikamente nur bei fortgeschrittener Krankheit vergütet. Eine neue Studie schätzt die Kosten verschiedener Therapien von Hepatitis C bis 2030 ab. Der Befund: Wird die neuste Medikamentenklasse nur für ausgewählte Patienten eingesetzt, zieht das erhebliche Folgekosten bei nicht optimal behandelten Patienten nach sich, die sich bei frühzeitiger Behandlung mit DAA-Medikamenten vermeiden liessen.

(interpharma)

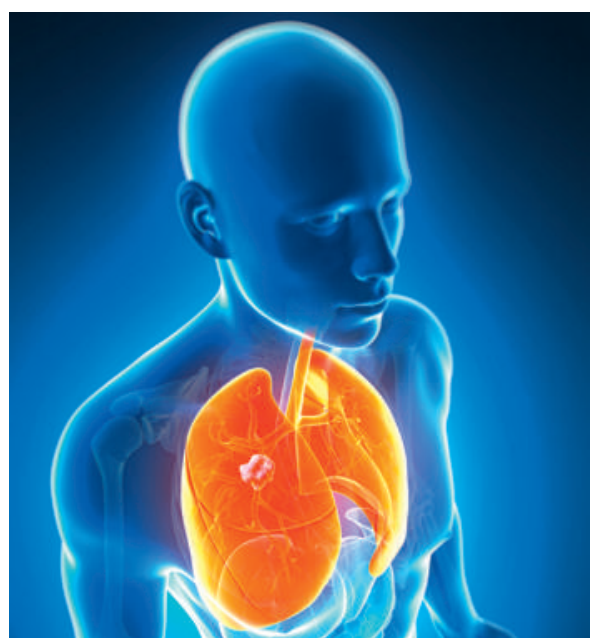

Le cancer des poumons est le plus mortel depuis plus de 40 ans.

\section{Neuer Blutgerinnungshem- mungs-Ausweis für Patienten}

Die Schweizerische Herzstiftung hat zusammen mit der Schweizerischen Gesellschaft für Kardiologie SGK einen umfassenden Gerinnungshemmungs-Ausweis entwickelt. Der Ausweis deckt neu alle Arten der oralen Antikoagulation und Thrombozytenaggregationshemmung ab. Der praktische Ausweis unterstützt Patientinnen und Patienten beim Selbstmanagement der Blutgerinnung und dient zur Erfassung der zu kontrollierenden Werte. Er enthält die persönlichen Angaben zur Indikation und Therapie, darüber hinaus auch wichtige Informationen im Zusammenhang mit der Behandlung sowie zu möglichen Gefahren. Die Liste mit einer zwölfmonatigen INR-Kontrolle lässt sich heraustrennen, falls der Patient keine Verlaufskontrolle benötigt. Der neue Ausweis (Format A6) ist ab sofort in den Sprachen Deutsch, Französisch und Italienisch bei der Schweizerischen Herzstiftung erhältlich.

(Schweizerische Herzstiftung)

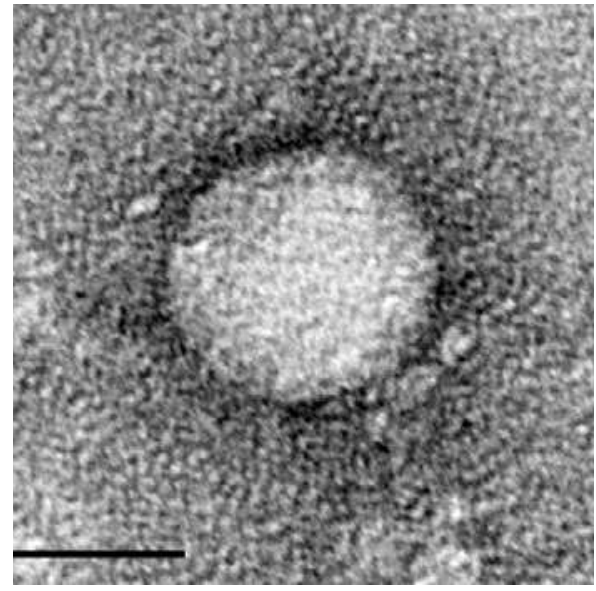

Hoffnung für Hepatitis-C-Patienten:

Neue Therapien stehen zur Verfügung.

\section{Une personne sur vingt meurt du cancer des poumons}

La population résidante de la Suisse a enregistré 64961 décès en 2013. Les maladies cardiovasculaires représentent avec un tiers des décès les principales causes de décès depuis des décennies. Leur part ne cesse toutefois de se réduire: le risque de mourir d'une maladie cardiovasculaire a donc presque diminué de moitié en 18 ans. Le cancer reste la deuxième cause de décès. Le cancer des poumons est de loin celui qui tue le plus depuis plus de 40 ans. Il a causé la mort de près de 2000 hommes et de plus de 1200 femmes en 2013. Ces 18 dernières années, il a baissé de $32 \%$ chez les hommes mais a augmenté de $47 \%$ chez les femmes.

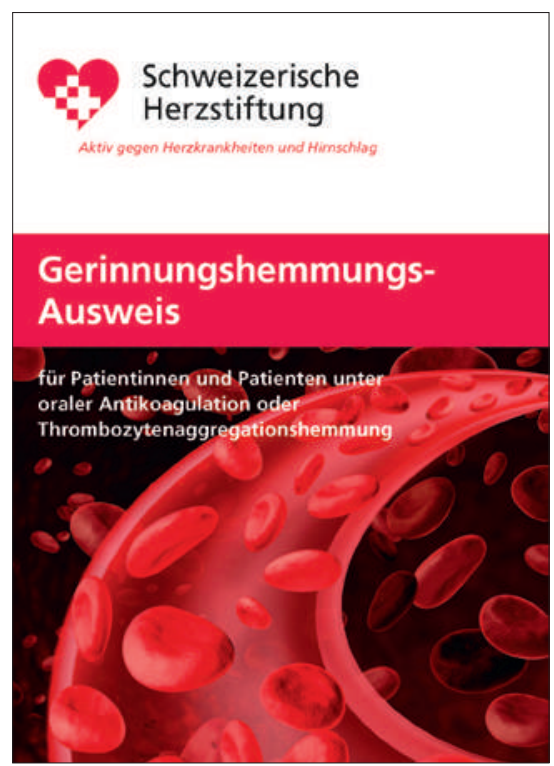

Unterstützt Betroffene beim Selbstmanagement der Blutgerinnung und bei der Kontrolle der Werte: der neue Blutgerinnungshemmungs-Ausweis.
Weniger Sackgassen für Fussgänger

Wer möglichst oft zu Fuss unterwegs ist, tut seiner Gesundheit viel Gutes. Seit kurzem hilft das Verkehrssignal "Sackgasse mit Ausnahmen», vermeintliche Sackgassen als mögliche Fussgängerwege zu enttarnen. Das neue Signal kennzeichnet Strassen und Wege, die für den Fuss- und/oder den Veloverkehr durchlässig sind. Diese Information musste bisher mit einer Zusatztafel kommuniziert werden. Mit der jüngst in Kraft getretenen revidierten Signalisationsverordnung ist das neue Signal, an dessen Erarbeitung und Evaluation Fussverkehr Schweiz vor zehn Jahren massgeblich mitgewirkt hat, nun offiziell.

(Fussverkehr Schweiz)

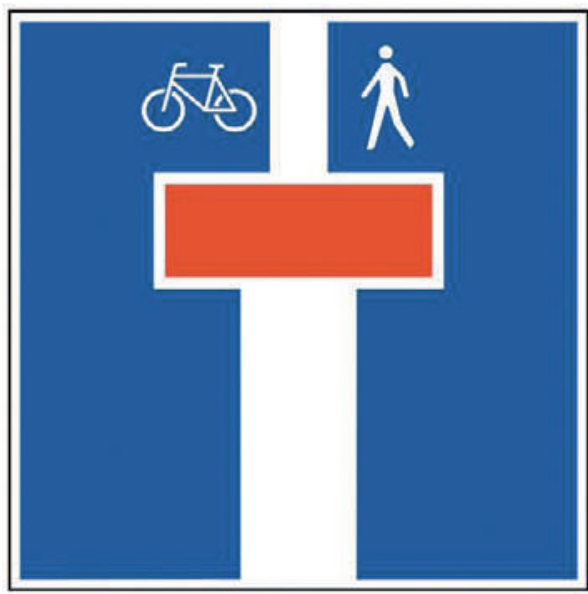

Nicht immer eine Sackgasse: Manchmal sind vermeintlich unpassierbare Wege auch für Fuss gänger und Fahrradfahrer durchlässig.

Prix Croix-Rouge pour Point d'Eau Lausanne Décerné pour la cinquième fois, le Prix CroixRouge de la Croix-Rouge suisse (CRS) récompensant des actions particulièrement louables dans le domaine humanitaire revient cette année à la fondation Point d'Eau Lausanne (PEL). PEL propose des prestations à bas seuil dans les domaines de l'hygiène, de la santé et d'orientation par des conseils sociaux. II accueille des personnes démunies ou défavorisées, sans distinction d'âge, de nationalité, de religion, de sexe ou de statut légal en Suisse. La grande majorité des 32000 prestations fournies chaque année aux plus démunis sont assurées par quelque 150 bénévoles. En remettant à la fondation le Prix CroixRouge, doté de $30000 \mathrm{CHF}$, la CRS récompense un engagement exemplaire en faveur de l'intégration sociale des personnes défavorisées. 